\title{
Minimum Length Scheduling for Discrete Rate Based Full Duplex Wireless Powered Communication Networks ${ }^{\star}$
}

\author{
Muhammad Shahid Iqbal ${ }^{1}$, Yalcin Sadi ${ }^{2}$, and Sinem Coleri Ergen ${ }^{1}$ \\ 1 Electrical and Electronics Engineering, \\ Koc University, Istanbul 34450, Turkey \\ \{miqbal16, sergen\}@ku. edu.tr \\ 2 Electrical and Electronics Engineering, \\ Kadir Has University, Istanbul 34083, Turkey \\ \{yalcin.sadi\}@khas.edu.tr
}

\begin{abstract}
In this study, we consider a wireless powered communication network where multiple users with radio frequency energy harvesting capabilities communicate to a hybrid energy and information access point in full duplex mode. We characterize an optimization framework for minimum length scheduling to determine the optimal rate adaptation and transmission scheduling subject to energy causality and traffic demand constraints of the users considering discrete-rate transmission model. We first formulate the problem as a mixed integer nonlinear programming problem which is hard to solve for a global optimum in polynomial-time. Then, based on an analysis on the characteristics of the optimal solution, we derive optimality conditions for rate adaptation and scheduling using which we propose a fast polynomial-time complexity heuristic algorithm. We illustrate through numerical analysis that the proposed algorithm performs very close to optimal for various network scenarios.
\end{abstract}

Keywords: Energy harvesting - Wireless powered communication networks · Rate adaptation · Scheduling · Optimization.

\section{Introduction}

Due to long range, full control on energy transfer, and small circuitry, Radio Frequency $(\mathrm{RF})$ based energy transfer is a preferred choice to replenish the batteries of the nodes once they are depleted. Wireless powered communication networks (WPCNs) are becoming very popular for the low power networks in which a dedicated hybrid access point (HAP) transmits a continuous energy for the users in the downlink and users harvest this energy for their data transmission in the uplink. WPCN have been studied in many different scenarios and considering different system models with the objective of total transmission time minimization and throughput maximization. Harvest-then-transmit is the first protocol in

\footnotetext{
* This work is supported by Scientific and Technological Research Council of Turkey
} Grant \#117E241. 
which the whole frame length is divided into two non-overlapping time durations dedicated for power and data transmission respectively and the data transmission interval is further divided into non overlapping slots allocated to each user [5] to maximize the sum throughput. The same system model is used to minimize the total transmission time in [1]. For throughput maximization, due to higher energy harvesting rates and low transmit power requirements, the near user dominate in the schedule which results in a doubly near far phenomenon. This unfair resource allocation motivated the authors for different objectives such as minimum throughput maximization [8], weighted sum throughput maximization [2] and common throughput maximization [6]. The authors in $[5,1$, $8,2,6]$ have considered a half duplex WPCN model where all users have equal time for energy harvesting and hence the transmission order is not important. Furthermore, all of these models have considered continuous transmission rate models without a user transmit power constraint considering a simplistic model.

Some of the studies have recently started incorporating the full duplex technique for WPCN in which users can perfom simultaneous energy harvesting and data transmission. For the full duplex model, [7] have presented the work for total time minimization in which the HAP is operating in full duplex mode and users are half duplex while [4] have considered full duplex users. In full duplex, users can harvest energy during both their own transmissions and the transmission of previously scheduled users which results in an uneven energy harvesting time for each user and hence making scheduling critical for the total time minimization. The authors in $[3,9]$ have paid attention to scheduling but in a limited context, such as in [3], the authors have used Hungarian algorithm to schedule the users with an objective of total transmission time minimization. However, for such sequence dependent networks, Hungarian algorithm requires exponential computational complexity. On the other hand, authors in [9] have divided the whole frame into a constant number of equal length time slots allocated to the users yielding underutilization of the resources. Moreover, all existing studies have considered continuous rate transmission model; whereas, discrete rate transmission model is missing for minimum length scheduling which will be the main focus of this paper.

The goal of this paper is to determine optimal time allocation, rate adaptation and scheduling with the objective of minimizing the schedule length subject to the traffic requirements of the users, the maximum transmit power constraint, and the energy causality constraint considering initial battery levels and a realistic energy harvesting model, in an in-band full-duplex WPCN.

The original contributions of this paper are listed as follows:

- We characterize the minimum length scheduling problem for a discrete rate transmission model based full duplex WPCN and mathematically formulate as a mixed integer non-linear programming problem.

- In order to solve the problem fast and efficiently, we propose a polynomial time complexity scheduling algorithm based on an analysis on the optimality conditions of the problem. 


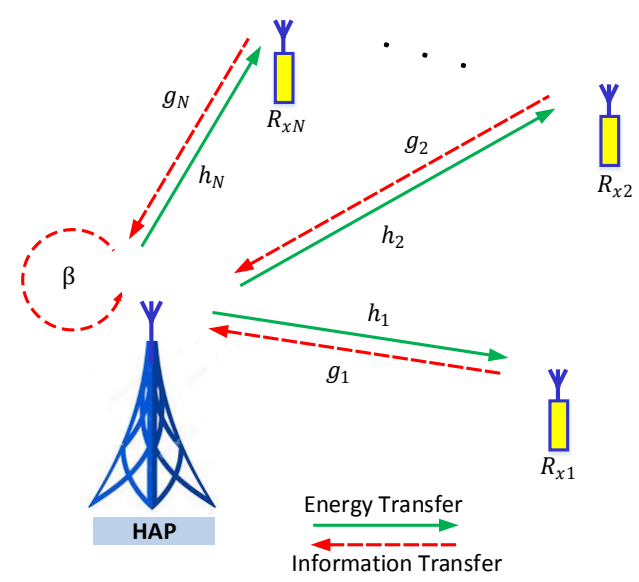

Fig. 1. Architecture of Wireless Powered Communication Network

- We illustrate the performance of the proposed algorithm in comparison to a predetermined transmission order based heuristic algorithm and to the optimal solution.

The rest of the paper is organized as follows. The system model and assumptions are given in Section 2. The optimization problem for minimum length scheduling is presented in Section 3. In Section 4, we introduce the proposed scheduling algorithm. The numerical results are provided and discussed in Section 5. Finally, Section 6 presents the concluding remarks.

\section{System Model and Assumptions}

The system model and assumptions are described as follows:

1. The WPCN architecture, as depicted in Fig. 1, consists of a HAP and N users; i.e., sensors or machine type communications (MTC) devices. Both the users and the HAP are equipped with a full-duplex antenna. Full duplex antennas are used for simultaneous wireless energy transfer and data transmission on downlink and uplink channels, respectively. The channel gains for the downlink and uplink channels are assumed to be different. The downlink channel gain from the HAP to user $i$ is denoted by $h_{i}$. The uplink channel gain from user $i$ to the HAP is denoted by $g_{i}$. Both downlink and uplink channels are assumed to be block-fading, i.e., the channel gains remain constant over the scheduling frame. We assume that the HAP has perfect channel state information (CSI); i.e., the channel gains are perfectly known at the HAP. 
2. The HAP has a stable power connection and continuously radiates a power $P_{h}$. On the other hand, the users are completely dependent on the harvested energy and no other supply is available for data transmission. All users harvest energy from the HAP and the harvested energy is stored in a battery with a large enough capacity such that no overflow will occur. The initial battery level is denoted by $B_{i}$ which is the amount of energy already stored in the battery at the start of the transmission cycle.

3. Time division multiple access protocol is used as the medium access protocol for the uplink data transmission from the users to the HAP. Time is partitioned into possibly variable length scheduling frames and then each frame is further divided into variable length time slots for the user allocation.

4. The energy harvesting rate of user $i$, denoted by $C_{i}$, depends on the antenna efficiency $\eta_{i}$, downlink channel $h_{i}$ and the power transmitted by the HAP $P_{h}$ as follows:

$$
C_{i}=\eta_{i} h_{i} P_{h}
$$

5. We assume user $i$ has a traffic demand $D_{i}$ bits to be transmitted over the scheduling frame.

6. We use discrete rate transmission model, in which a finite set of rates $r=$ $\left(r^{1}, r^{2}, \cdots, r^{M}\right)$ and a finite set of SINR levels $\gamma=\left(\gamma^{1}, \gamma^{2}, \cdots, \gamma^{M}\right)$ are determined such that user $i$ can transmit at rate $r^{k}$ successfully in the allocated time slot if the SINR achieved for user $i$ is

$$
\gamma_{i}=\frac{P_{i} g_{i}}{\sigma_{o}^{2}+\beta P_{h}} \geq \gamma^{k}
$$

where the term $\beta P_{h}$ is the power of self interference at the HAP and $\sigma_{o}^{2}$ is the noise power.

7. We use continuous power model in which the transmission power of a user can take any value below a maximum level $P_{\max }$, which is imposed by the regulatory authorities to avoid the interference to nearby systems.

\section{Minimum Length Scheduling Problem}

In this section, we introduce the Discrete Rate based Minimum Length Scheduling Problem, referred as DR-MLSP.

The joint optimization of the time allocation, power control, rate adaptation and scheduling with the objective of minimizing the schedule length is formulated as follows: 
DR-MLSP:

$$
\begin{array}{ll}
\text { minimize } & \sum_{i=0}^{N} \tau_{i} \\
\text { subject to } \quad & B_{i}+C_{i} \tau_{0}+C_{i} \sum_{j=1}^{N} a_{i j} \tau_{j}-P_{i} \tau_{i} \geq 0, \\
& P_{i} \leq P_{\max }, \\
& \tau_{i} \chi_{i} \geq D_{i}, \\
& \chi_{i}=\sum_{k=1}^{M} z_{i k} r^{k}, \\
& P_{i} g_{i}-\left(\sum_{k=1}^{M} z_{i k} \gamma^{k}\right)\left(\sigma_{o}^{2}+\beta P_{h}\right) \geq 0, \\
& \sum_{k=1}^{M} z_{i k}=1, \\
& a_{i j}+a_{j i}=1, \\
& P_{i} \geq 0, \tau_{i} \geq 0, a_{i j} \in\{0,1\}, z_{i k} \in\{0,1\} .
\end{array}
$$

The variables of the problem are $P_{i}$, the transmit power of user $i ; \tau_{i}$, the transmission time of user $i$; $a_{i j}$, a binary variable that takes value 1 if user $i$ is scheduled before node $j$ and 0 otherwise; and $z_{i k}$, a binary variable which takes value 1 if user $i$ is allocated rate $r^{k}$ and 0 otherwise. In addition, $\tau_{0}$ denotes an initial waiting time duration during which all users only harvest energy without transmitting any information.

The objective of the optimization problem is to minimize the schedule length as given by Equation (3a). Equation (3b) gives the energy causality constraint: The total amount of available energy, including both the initial energy and the energy harvested until and during the transmission of a user, should be greater than or equal to the energy consumed during its transmission. Equation (3c) represents the maximum transmit power constraint. Equation (3d) represents the traffic demand constraint of the users where $\chi_{i}$ denotes the transmission rate of user $i$ as given by Equation (3e). Equation (3f) represents the SINR constraint of the users as presented in Equation (2). Equation (3g) represents the rate adaptation; i.e., user can pick a single transmission rate from the specified finite set. Finally, Equation (3h) represents the transmission order constraint for the users.

The optimization problem formulation presented in Equations (3) is a Mixed Integer Non-Linear Programming (MINLP) problem which is generally hard to solve for a global optimum in polynomial-time. On the other hand, one straightforward solution is a brute-force search algorithm that enumerates all possible transmission orders and rate adaptations among which the one with minimum corresponding schedule length is determined as the optimal solution. However, 
this exact solution is intractable even for medium network sizes. In the following section, we propose a polynomial-time complexity algorithm based on the analysis on the optimality conditions of the DR-MLSP.

\section{Scheduling Algorithm}

In this section, we first analyze the optimality conditions for the DR-MLSP and then present a polynomial-time complexity algorithm.

We start by investigating the optimal rate adaptation policy for a single user. Initially, a user may not be able to transmit with even the minimum possible transmission rate level $r^{1}$ since the initial energy available $B_{i}$ may not be able to support the corresponding transmit power that will satisy the SNIR constraint given by Equation (2). Each transmission rate level $r^{k}$ requires certain amount of energy available to complete the required data transmission for the user. Let $t_{i}^{k}$ be the first time instant at which user $i$ can afford to use transmission rate $r^{k}$ via satisfying the SNIR constraint $\gamma_{i} \geq \gamma^{k}$ using the harvested energy. Note that $t_{i}^{1} \leq t_{i}^{2} \leq \ldots \leq t_{i}^{M}$.

Consider Figure 2 illustrating the transmission completion (end time) vs. allocation time (start time) for a user $i . \tau_{0}$ denotes the initial waiting time for user $i$ to be able to transmit with $r^{1}$ by satisfying $\gamma_{i} \geq \gamma^{1}$. Then, for a finite duration, user $i$ can only transmit with $r^{1}$; i.e., $r^{1}$ region. At $t_{i}^{2}$, user $i$ can support $r^{2}$ for the first time and $r^{2}$ is the maximum rate it can support for a specific duration; i.e., $r^{2}$ region. Figure illustrates regions for the transmission rate values $r^{k}$ s. Let $s_{i}$ be the start time of the transmission of user $i$. Then, the completion time of the transmission is given by $e_{i}=s_{i}+\tau_{i}$ where $\tau_{i}$ is the transmission time of user $i$ such that $\tau_{i}=D_{i} / \chi_{i}$. Since, user $i$ can support a higher transmission rate level at $t_{i}^{k}$ instants, $e_{i}$ is not a monotonically increasing function of $s_{i}$. At any $s_{i}=t_{i}^{k}$ value, $e_{i}$ decreases discontinuously by an amount of $D_{i} / r^{k-1}-D_{i} / r^{k}$ since the transmission rate jumps from $r^{k-1}$ to $r^{k}$. This suggests that waiting for the next transmission rate may decrease the completion time for a single user depending on the time instant at which the scheduling decision is given. In the following, we illustrate the optimal rate adaptation and scheduling policy for a single user.

We first start by the following definition.

Definition 1. Let $t_{i}^{\text {dec }}$ is the time instant at which a scheduling decision is made for a user $i$. Then, let $s_{i}^{*} \geq t_{i}^{\text {dec }}$ be the starting time for user $i$ yielding the minimum completion time $e_{i}^{*}$ such that $e_{i}^{*}=\min _{s_{i} \geq t_{i}^{\text {dec }}} s_{i}+\tau_{i}\left(s_{i}\right)$. Minimum length scheduling (MLS) slot for user $i$ at time $t_{i}^{\text {dec }}$ is then defined as the time slot for user $i$ allocated in the interval $\left[s_{i}^{*}, e_{i}^{*}\right]$ where $s_{i}^{*} \geq t_{i}^{\text {dec }}$.

Then, it is evident that for a single user, the DR-MLSP problem is solved by allocation of MLS slot at $t_{i}^{d e c}=0$. Figure 2 illustrates the MLS slot for a single user $i$. Note that the MLS slot for user $i$ starts at $t_{i}^{2}$ where user $i$ can afford rate $r^{2}$ for the first time. This suggests that even if user $i$ can transmit with $r^{1}$ previously, the optimal policy is to wait until the time instant $t_{i}^{2}$ since 


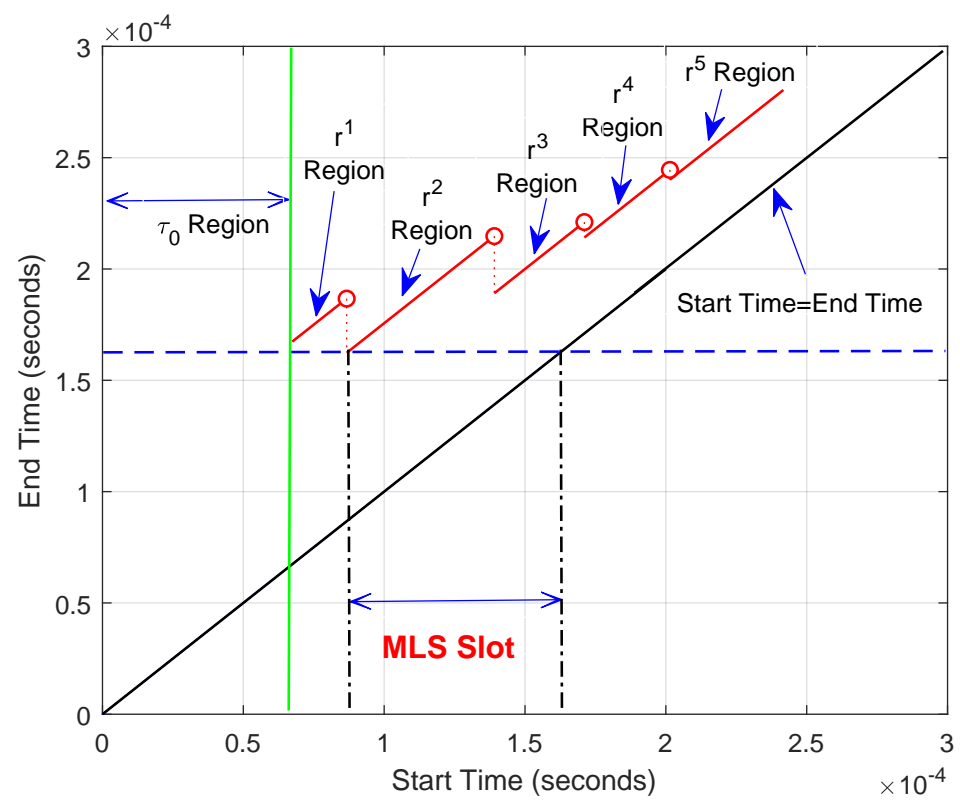

Fig. 2. Illustration of Rate Regions and MLS Slot

the decrease in the transmission time due to this rate increase is larger than the waiting duration. Note that MLS slot for the user starts at a rate change instant $t_{i}^{k}$ which is not a coincidence for this specific scenario. The following lemma illustrates this behaviour.

Lemma 1. $M L S$ slot for user $i$ at $t_{i}^{\text {dec }}$ starts at either $t_{i}^{\text {dec }}$ or $t_{i}^{k} \geq t_{i}^{\text {dec }}$ for some $k \in[1, M]$.

Proof. Let starting time for the MLS slot of user $i$ be $s_{i}^{*}$ such that $s_{i}^{*} \neq t_{i}^{\text {dec }}$ or $s_{i}^{*} \neq t_{i}^{k}$ for $k \in[1, M]$. Then, let the completion time be $e_{i}^{*}$. Suppose that $s_{i}^{*}$ is inside $r^{l}$ region; i.e., $t_{i}^{l}<s_{i}^{*}<t_{i}^{l+1}$. If $t_{i}^{\text {dec }} \leq t_{i}^{l}$, then starting the transmission time of user $i$ at $t_{i}^{l}$ decreases the completion time by $s_{i}^{*}-t_{i}^{l}$ since the transmission rate remains the same within $r^{l}$ region. If $t_{i}^{d e c}>t_{i}^{l}$, then starting the transmission time of user $i$ at $t_{i}^{d e c}$ decreases the completion time by $s_{i}^{*}-t_{i}^{d e c}$. This is a contradiction by definition of MLS slot.

Lemma 1 illustrates that for a single user, the optimal scheduling policy is allocation of the user at either the time instant where the scheduling decision is made or at one of the time instants where the transmission rate changes for the user. Then, MLS slot determination can be made by evaluating at most $M+1$ time instants where $M$ is the number of rate levels. Furthermore, Lemma 1 shows that for a predetermined transmission order of users, the optimal scheduling policy is simply determining the MLS slots for the users in the predetermined order. 


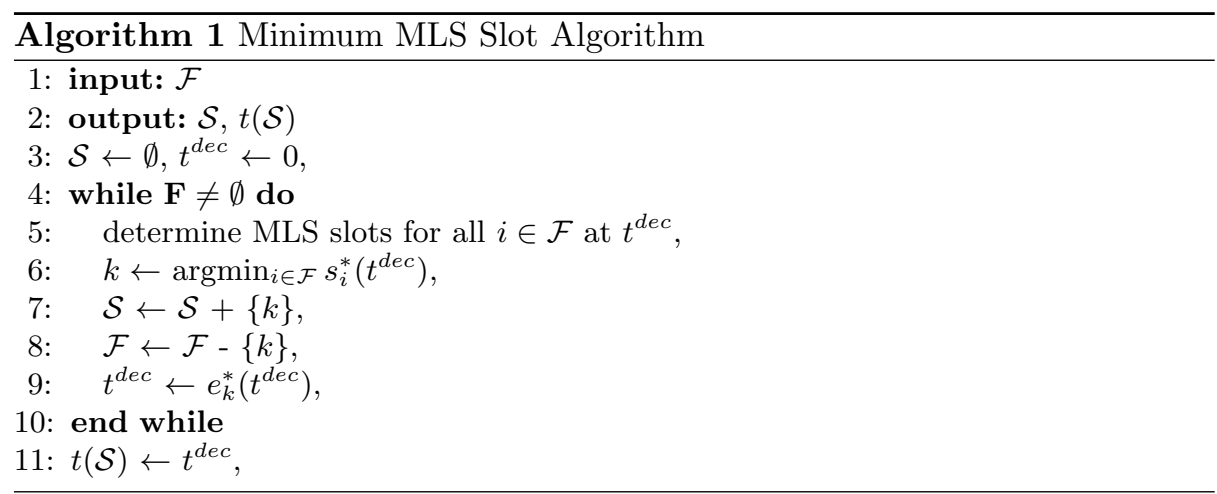

In the following, we propose a polynomial-time algorithm based on the foregoing discussion.

The Minimum MLS Slot Algorithm (MMSA), given in Algorithm 1, is described next. Input of MMSA algorithm is a set of users, denoted by $\mathcal{F}$, with the characteristics specified in Section 2 (Line 1). The algorithm starts by initializing the schedule $\mathcal{S}$ where the $i^{t h}$ element of $\mathcal{S}$ is the index of the user scheduled in the $i^{t h}$ time slot and the scheduling decision time $t^{\text {dec }}$ (Line 3). At each step of the algorithm, MMSA determines the MLS slots for the unallocated users at $t^{\text {dec }}$ (Line 5), and picks the user with the minimum MLS slot starting time $s_{i}^{*}$ (Line $6)$. Then, this user is allocated to its MLS slot starting at $s_{k}^{*}$ and completed at $e_{k}^{*}$ (Lines $\left.7-8\right)$. Algorithm continues by updating the scheduling decision time (Line 9) and giving scheduling decisions for the remaining users (Lines $4-10$ ). MMSA terminates when all users in $\mathcal{F}$ are scheduled and outputs the schedule $\mathcal{S}$ with schedule length $t(\mathcal{S})$ (Line 11).

\section{Performance Evaluation}

The goal of this section is to evaluate the performance of the proposed algorithm MMSA in comparison to the optimality and a predetermined transmission order based heuristic algorithm, denoted by PDO. PDO aims at minimizing the schedule length for a given transmission order of the users by allocating each user as early as possible, without considering scheduling. The optimal solution is obtained by a brute force algorithm, denoted by BFA, enumerating all possible transmission orders and then picking the best schedule with minimum length.

\subsection{Simulation Setup}

Simulation results are obtained by averaging over 1000 independent random network realizations. The attenuation of the links considering large-scale statistics are determined using the path loss model given by

$$
P L(d)=P L\left(d_{0}\right)+10 \alpha \log _{10}\left(\frac{d}{d_{0}}\right)+Z
$$




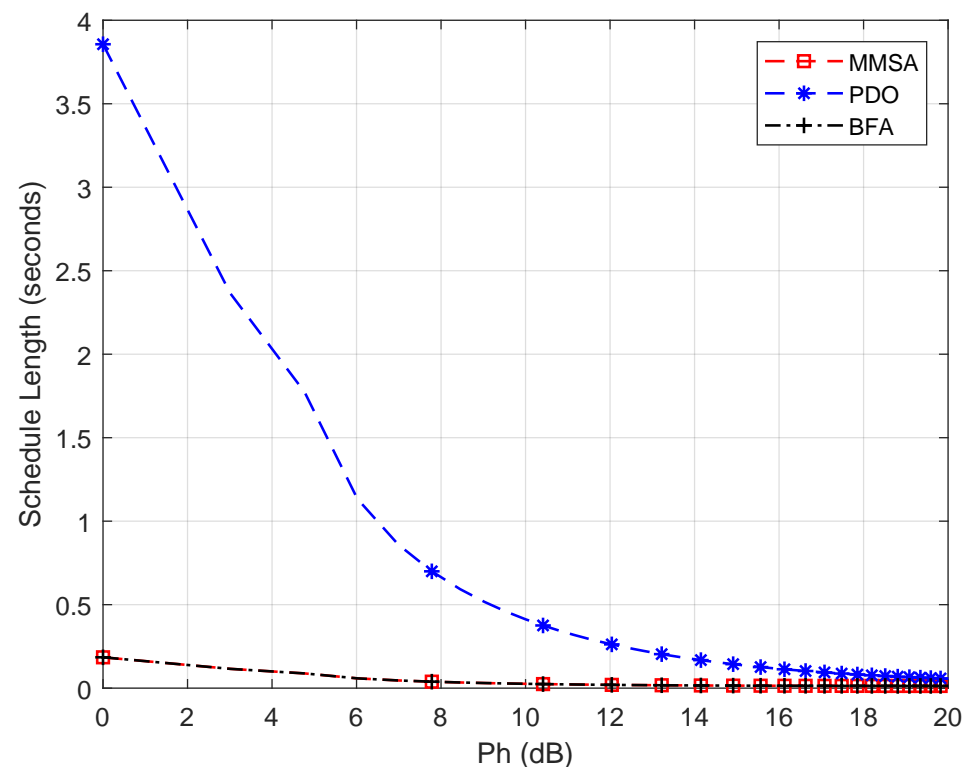

Fig. 3. Schedule Length vs. HAP trasmit power $P_{h}$

where $P L(d)$ is the path loss at distance $d$ in $d B, d_{0}$ is the reference distance, $\alpha$ is the path loss exponent, and $Z$ is a zero mean Gaussian random variable with standard deviation $\sigma$. The small-scale fading has been modelled by using Rayleigh fading with scale parameter $\Omega_{i}$ set to mean power level obtained from the large-scale path loss model. The parameters used in the simulations are $\eta_{i}=1$ for $i \in[1, N] ; D_{i}=100$ bits for $i \in[1, N] ; W=1 \mathrm{MHz} ; d_{0}=1 \mathrm{~m}$; $P L\left(d_{0}\right)=30 \mathrm{~dB} ; \alpha=2.76, \sigma=4$. The self interference coefficient $\beta$ is taken as $-70 \mathrm{dBm}$. We use $M=5$ discrete rate and corresponding SNIR levels.

\subsection{Scheduling Performance}

Fig. 3 illustrates the performance of the proposed algorithm for different values of the transmit power of the HAP. Figure illustrates that the proposed algorithm performs very close to the optimal solution. MMSA outperforms PDO significantly for a wide range of $P_{h}$. It can be observed that for low values of $P_{h}$, scheduling is more critical because the users with low energy require more time to achieve a particular rate level. However, for higher values of the $P_{h}$ the users can harvest energy at a higher rate so they can reach to the desired SNR levels quickly hence less waiting time is required. Therefore, the superiority of MMSA over PDO is really significant for low values of $P_{h}$ whereas the performance improvement decreases as $P_{h}$ increases.

Fig. 4 illustrates the performance of MMSA for different number of users. Note that due to exponential complexity of the brute force enumeration used in 


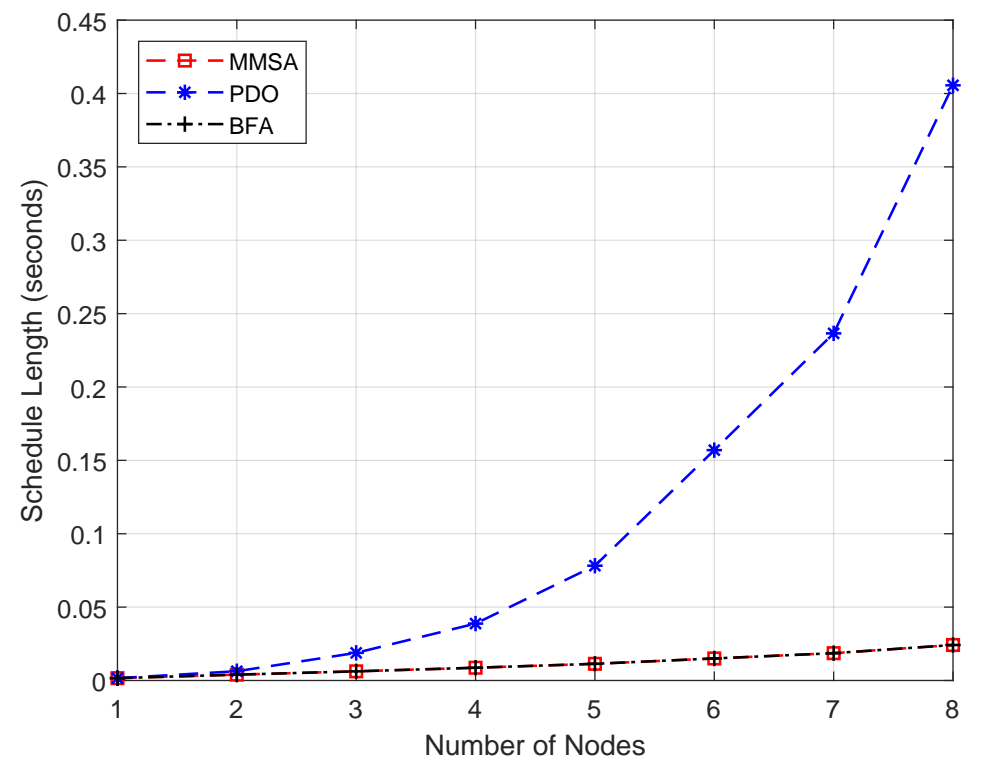

Fig. 4. Schedule Length vs. Number of nodes

BFA, simulation results are obtained up to 8 users. Similar to Fig. 3, MMSA performs almost optimal while outperforming PDO significantly. As the number of users increases, scheduling becomes more critical since an arbitrary transmission order may result in delays in the transmission of high rate users even if these users do not need large energy harvesting durations. Moreover, increasing number of users puts an arbitrary transmission order further away from the optimality hence increasing the suboptimality of PDO. On the other hand, if the users are scheduled properly by eliminating the unnecessary waiting intervals for achieving higher rates as in MMSA, the optimality performance is preserved showing the robustness of MMSA to the network size. Note that robustness to the network size is very important for future networks with high number of machine type devices or sensors.

Finally, Fig. 5 illustrates the behaviour of the network for different required SNR levels for minimum rate transmissions. It can be observed that for lower values of SNR, the users can start transmission earlier since the amount of energy required for that SNR value will be harvested earlier. This yields smaller schedule length. On the other hand, as the required SNR increases, users need to wait longer to harvest enough energy and achieve successful transmissions increasing the overall schedule length. MMSA performs again close to optimal while significantly outperforming PDO illustrating the importance of a proper scheduling decision. 


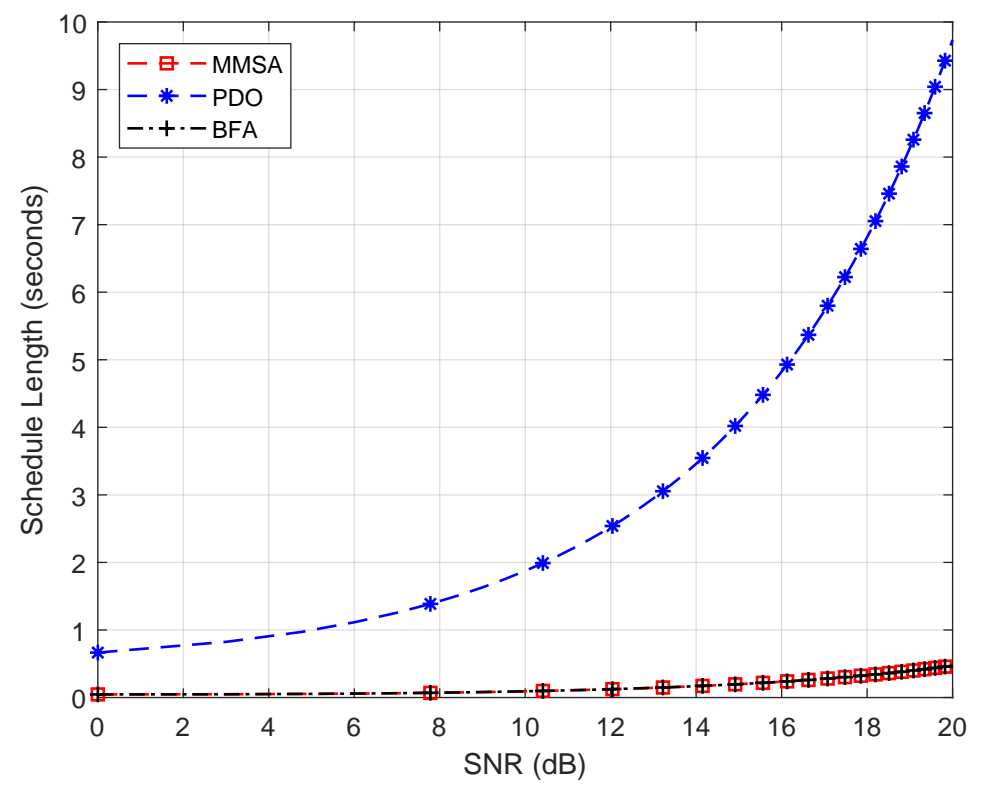

Fig. 5. Schedule Length vs SNR values

\section{Conclusion and Future Work}

In this paper, we have investigated minimum length scheduling problem considering discrete rate transmission model in a full duplex wireless powered communication network. We have characterized an optimization framework to determine the optimal time allocation, rate adaptation and scheduling subject to maximum transmit power, traffic demand and energy causality requirements of the users. First we have mathematically formulated the problem as a mixed integer nonlinear programming problem which is difficult to solve for the global optimum in polynomial time. In order to solve the problem fast and efficiently, then, we have proposed a polynomial time algorithm based on an optimality analysis on the optimization problem. Through numerical analysis, we have shown that the proposed algorithm performs almost optimal while outperforming a predetermined order based heuristic algorithm significantly.

For future work, we plan to extend this work to propose optimal polynomialtime algorithm for the investigated system model. Besides, we aim at extending the system model incorporating multiple hybrid access points and beamforming technology.

\section{References}

1. Chi, K., Zhu, Y., Li, Y., Huang, L., Xia, M.: Minimization of transmission completion time in wireless powered communication networks. IEEE Internet of Things Journal 4(5), 1671-1683 (Oct 2017). https://doi.org/10.1109/JIOT.2017.2689777 
2. Di, X., Xiong, K., Fan, P., Yang, H., Letaief, K.B.: Optimal resource allocation in wireless powered communication networks with user cooperation. IEEE Trans. on Wireless Commun. 16(12), 7936-7949 (Dec 2017)

3. Hu, J., Xue, Y., Yu, Q., Yang, K.: A joint time allocation and ue scheduling algorithm for full-duplex wireless powered communication networks. In: 2017 IEEE 86th Vehicular Technology Conference (VTC-Fall). pp. 1-5 (Sep 2017)

4. Ju, H., Chang, K., Lee, M.: In-band full-duplex wireless powered communication networks. In: 2015 17th Int. Conf. on Advanced Commun. Technology (ICACT). pp. 23-27 (July 2015)

5. Ju, H., Zhang, R.: Throughput maximization in wireless powered communication networks. In: 2013 IEEE Global Commun. Conference (GLOBECOM). pp. 40864091 (Dec 2013)

6. Ju, H., Zhang, R.: Throughput maximization in wireless powered communication networks. IEEE Trans. on Wireless Commun. 13(1), 418-428 (January 2014)

7. Kang, X., Ho, C.K., Sun, S.: Full-duplex wireless-powered communication network with energy causality. IEEE Trans. on Wireless Commun. 14(10), 5539-5551 (Oct 2015)

8. Liu, L., Zhang, R., Chua, K.: Multi-antenna wireless powered communication with energy beamforming. IEEE Trans. on Commun. 62(12), 4349-4361 (Dec 2014)

9. Pathak, K., Kalamkar, S.S., Banerjee, A.: Optimal user scheduling in energy harvesting wireless networks. IEEE Trans. on Commun. 66(10), 4622-4636 (Oct 2018) 